\title{
DISPOSICIÓN HACIA EL PENSAMIENTO CRÍTICO EN ESTUDIANTES DE UNA UNIVERSIDAD ESTATAL DE LIMA METROPOLITANA
}

\author{
Ana Esther Delgado VÁsquez \\ https://orcid.org/0000-0002-5649-1262 \\ LUIS Miguel ESCURRA MAYAUTE \\ https://orcid.org/0000-0002-3015-9659 \\ María Clotilde Atalaya Pisco \\ https://orcid.org/0000-0003-3266-9692 \\ Juan Pequeña Constantino \\ https://orcid.org/0000-0002-6085-1099 \\ Rolando Santiago Solís Narro \\ https://orcid.org/0000-0002-5072-5558
}

DAVID MARCIAL ÁlVAREZ Flores

https://orcid.org/0000-0002-2245-106X

WILLIAM JesÚS TORRES ACUÑA

https://orcid.org/0000-0002-8050-9709

Abel Cuzcano Zapata

Esther Alicia Castro Celis

Rosa EMILIA Rodríguez TARAzOna

Universidad Nacional Mayor de San Marcos

Recibido: 15 de julio del 2019 / Aceptado: 10 de setiembre del 2019

doi: 10.26439/persona2019.n022(2).4565

Resumen. Se investigó la disposición hacia el pensamiento crítico en estudiantes de diversas áreas profesionales, hombres y mujeres, de una universidad estatal de Lima Metropolitana. Participaron 1376 universitarios, obtenidos a través de un procedimiento de muestreo no probabilístico por cuotas. Se realizaron las comparaciones considerando las diversas carreras profesionales y el sexo de los participantes. Los resultados indican que existen diferencias estadísticamente significativas solo en el área Curiosidad de la Disposición hacia el Pensamiento Crítico según las áreas profesionales de la universidad.

Palabras clave: disposición hacia el pensamiento crítico / estudiantes / universidad pública

\section{CRITICAL THINKING DISPOSITION AMONG STUDENTS OF A PUBLIC UNIVERSITY IN LIMA METROPOLITAN AREA}

\begin{abstract}
Critical thinking disposition was researched in male and female students from different professional areas of a public university in Lima Metropolitan Area. One thousand three hundred seventy-six $(1,376)$ university students were recruited using a non-probability quota sampling. Comparisons were made considering the participants' different professional areas and sex. The results show that there are only significant statistical differences in the Curiosity dimension of the test according to the professional areas of the university.
\end{abstract}

Keywords: critical thinking disposition / students / public university 


\section{INTRODUCCIÓN ${ }^{1}$}

Dewey (1933) planteaba que el pensamiento crítico constituía en el siglo pasado una de las más importantes metas educativas; sin embargo, no hubo un especial interés en que se desarrolle. Siegel (1990) señala que el interés por el pensamiento crítico surgió por varios motivos, entre ellos el hecho de observar que los estudiantes poseían muy pocas habilidades de pensamiento de orden superior, cuando es necesario que puedan pensar críticamente y sean capaces de participar de manera plena en la vida pública. Ennis (1986) señala que el pensamiento crítico se caracteriza por manejar y dominar las ideas y que su principal función es revisar las ideas, evaluarlas, repasar qué es lo que se entiende, procesar y comunicar mediante los otros tipos de pensamiento (lógico, matemático, verbal, etcétera).

Autores como Ennis (1986, 2011); Facione (1990); Facione y Facione (1992); Facione, Giancarlo, Facione y Gainen (1995); Facione, Facione y Giancarlo (1997) y Facione, Facione, Giancarlo y Gainen (2000) han demostrado que el pensamiento crítico está formado por habilidades (componente cognoscitivo) y por disposiciones (componente motivacional). El componente cognoscitivo ha sido definido por Ennis $(1986,2011)$ como el pensamiento racional, reflexivo, que se interesa en qué hacer o qué creer. Constituye un complejo proceso de pensamiento que considera que la razón predomina sobre otras dimensiones del pensamiento (López, 2012). Çubukcu (2006) afirma que el pensamiento crítico es un proceso cognoscitivo eficaz, organizado y funcional que va a permitir a una persona comprender sus pensamientos, entender las opiniones de los demás y mejorar su disposición para expresarse.

El pensamiento crítico también incluye las disposiciones acerca de cómo decidir, qué hacer o creer lógicamente. Según Facione, Facione, Giancarlo y Gainen (2000), la disposición hacia el pensamiento crítico (componente motivacional) es el conjunto de atributos caracterológicos de una persona para valorar y emplear el pensamiento crítico, pues se refiere a la motivación interna para utilizar las habilidades del pensamiento crítico.

Boisvert (2004) señala que el pensamiento crítico es muy importante en la vida adulta, debido a que va a facilitar las elecciones personales porque las hace más claras, como por ejemplo en la orientación profesional, al adoptar un estilo de vida o al adquirir bienes materiales. Plantea que las razones por las cuales se debe enseñar el pensamiento crítico son que la sociedad lo exige, que es necesario asegurar el desarrollo socioeconómico global, que se necesita tener una producción más racional y que se debe contrarrestar la manipulación.

1 Ana Esther Delgado Vásquez: adelgadov1@unmsm.edu.pe. El estudio fue patrocinado por el Vicerrectorado de Investigación y el Consejo Superior de Investigaciones (CSI) de la UNMSM, código N. ${ }^{\circ}$ A17180871 el año 2017. 
Se considera que la disposición al pensamiento crítico es una motivación interna consistente para enfrentar los problemas y tomar decisiones, haciendo uso del pensamiento (Escurra y Delgado, 2008b).

Según Facione, Facione, Giancarlo y Gainen (2000), Escurra y Delgado (2008a, b) y Velásquez y Figueroa (2010), las dimensiones de la disposición hacia el pensamiento crítico son las siguientes: búsqueda de la verdad, amplitud mental, capacidad de análisis, sistemático, confianza en el razonamiento, curiosidad y madurez para formular juicios.

León (2014) señala que los resultados de diversas investigaciones evidencian que existen ciertas disposiciones del pensamiento crítico que son rasgos medibles que van a influir en el desempeño de una persona como pensador crítico. García, Graterol y Triviño (2014) consideran que estas son esenciales para poner en práctica el buen pensamiento. Señalan que muchas veces se enseña a pensar, pero poniendo énfasis en el desarrollo de aptitudes y habilidades de pensamiento específicas, y que los estudiantes suelen no poner en práctica el conocimiento y las habilidades que han aprendido. Los autores consideran que lo mejor es combinar ambos enfoques, es decir, el desarrollo de las aptitudes con el cultivo de las disposiciones del pensamiento. De lo planteado surge la siguiente interrogante: ¿difiere la disposición hacia el pensamiento crítico entre los estudiantes de diversas áreas profesionales hombres y mujeres de una universidad estatal de Lima Metropolitana?

Los objetivos de este estudio han sido los siguientes:

\section{Objetivos generales}

1. Comparar la disposición hacia el pensamiento crítico entre los estudiantes de diversas áreas profesionales de una universidad estatal de Lima Metropolitana.

2. Comparar la disposición hacia el pensamiento crítico entre los estudiantes hombres y mujeres de una universidad estatal de Lima Metropolitana.

\section{Objetivos específicos}

1. Identificar la disposición hacia el pensamiento crítico de los estudiantes de diversas áreas profesionales de una universidad estatal de Lima Metropolitana.

2. Identificar la disposición hacia el pensamiento crítico de los estudiantes hombres y mujeres de una universidad estatal de Lima Metropolitana.

3. Comparar las distintas áreas de la disposición hacia el pensamiento crítico referidas a la búsqueda de la verdad, amplitud mental, capacidad de análisis, sistemático, confianza en el razonamiento, curiosidad y madurez para formular juicios entre los estudiantes de diversas áreas profesionales de una universidad estatal de Lima Metropolitana. 
4. Comparar las distintas áreas de la disposición hacia el pensamiento crítico referidas a la búsqueda de la verdad, amplitud mental, capacidad de análisis, sistemático, confianza en el razonamiento, curiosidad y madurez para formular juicios entre los estudiantes de una universidad estatal de Lima Metropolitana según el sexo.

En cuanto a las investigaciones internacionales sobre la disposición hacia el pensamiento crítico, se reportan algunas, tal como la de Velásquez y Figueroa (2010) quienes investigaron sobre el desarrollo del pensamiento crítico en estudiantes de las carreras universitarias de mayor demanda de El Salvador. Participaron 380 estudiantes de cuatro universidades de la zona metropolitana de San Salvador y una de Santa Tecla. Utilizaron CCTST 2000 de Facione, Facione y Winterhalter. Concluyeron que era necesario decidirse a formar personas con pensamiento crítico. Esta urgencia era particularmente importante y necesaria para los sistemas educativos de la región latinoamericana y, dentro de esta, para aquellos países con altos índices de dependencia económica, tecnológica, científica y cultural que requerían la innovación para la transformación.

Asimismo, Torres (2011) llevó a cabo un estudio acerca de la relación de la disposición en el desarrollo del pensamiento crítico y el aprendizaje de las Ciencias Naturales, en el que participaron treinta estudiantes de educación media de un colegio de la Ciudad de Tunja, Boyacá, con edades entre quince y dieciséis años. Se empleó una Escala de Conciencia y una entrevista que permitió a los estudiantes manifestar espontáneamente el concepto de disposición, su importancia en el proceso de formación y su relación con el aprendizaje de las Ciencias Naturales. Concluyó señalando que las disposiciones contribuían al desarrollo general del pensamiento; estas eran demostradas por los estudiantes tanto en el desarrollo de sus prácticas de aula como en sus prácticas contextuales, por lo tanto, era necesario que los profesores proporcionasen actividades y espacios que permitieran a los estudiantes el desarrollo de disposiciones positivas en su proceso de aprendizaje. La mayor parte de los participantes consideraba importantes las disposiciones positivas, pero señalaban que no desarrollaban actividades que pusieran en evidencia su utilidad. Los profesores debían ayudar a sus alumnos a desarrollar disposiciones de pensamiento estables y sólidas.

Del mismo modo, Bejarano, Galván y López (2014) investigaron sobre la motivación hacia el pensamiento crítico y el pensamiento crítico en 65 estudiantes de psicología de una institución de educación superior de Barrancabermeja. Se aplicó el PENCRISAL y la Escala de Motivación hacia el Pensamiento Crítico (EMPC). Se encontró que tanto los estudiantes varones como las mujeres presentaban debilidades importantes en su capacidad para pensar críticamente. Con relación a la motivación, los participantes reconocieron que el pensamiento crítico era importante y útil, pero era menor su disposición para utilizar este tipo de pensamiento y responsabilizarse de las exigencias que ello involucraba. 
En el Perú, Escurra y Delgado (2008a) llevaron a cabo la construcción de la Escala de Disposición hacia el Pensamiento Crítico en universitarios de la ciudad de Lima empleando el modelo de Samejima. Los participantes fueron 830 estudiantes de universidades estatales y particulares. Se concluyó que cada una de las áreas que constituyen la disposición hacia el pensamiento crítico se adecua al modelo de respuesta graduada de Samejima. Asimismo, el instrumento presentó evidencias de validez basadas en la estructura interna y confiabilidad por el método de consistencia interna.

Escurra y Delgado (2008b) investigaron acerca de la relación entre la disposición hacia el pensamiento crítico y los estilos de pensamiento en universitarios de Lima y Callao. Participaron 830 estudiantes. Los instrumentos utilizados fueron la Escala de Estilos de Pensamiento de Sternberg y la Escala de Disposición hacia el Pensamiento Crítico de Escurra y Delgado. Se encontraron correlaciones estadísticamente significativas entre las áreas del pensamiento crítico y los estilos de pensamiento. Asimismo, se hallaron diferencias estadísticamente significativas cuando se consideró el tipo de gestión de la universidad, el sexo de los participantes y el área profesional de los estudiantes, tanto en lo referente a los estilos de pensamiento como en lo que concernía a la disposición hacia el pensamiento crítico.

Perea (2017) estudió acerca de cómo se relacionaba la disposición hacia el pensamiento crítico y el rendimiento en el curso de Metodología de la Investigación en los estudiantes de una universidad privada de Lima Metropolitana. Participaron 263 universitarios de diversas facultades. Utilizó como instrumentos la Escala de Disposición hacia el Pensamiento Crítico de Escurra y Delgado y el promedio final del curso de Metodología de la Investigación. No encontró relación significativa entre la disposición hacia el pensamiento crítico y el rendimiento académico en el curso de Metodología de la Investigación. Encontró una relación baja, negativa pero estadísticamente significativa, entre el área curiosidad de la disposición hacia el pensamiento crítico y el rendimiento académico en el curso.

\section{MÉTODO}

\section{Participantes}

La población estuvo constituída por estudiantes varones y mujeres de diversas áreas profesionales de una universidad estatal de Lima Metropolitana. El tamaño de la muestra fue determinado considerando las características de la población. Para obtener la muestra se empleó un procedimiento de muestreo no probabilístico por cuotas, y una vez seleccionada la institución a ser incluida en el estudio, se estableció la proporción de estudiantes por área profesional y sexo, conservando la proporción de la población. Posteriormente se eligió a los participantes que fueron evaluados. 


\section{Instrumento}

El instrumento que se utilizó fue la Escala de Disposición hacia el Pensamiento Crítico de Escurra y Delgado (2008a), conformada por setenta ítems, los cuales se responden con un formato tipo likert de 7 puntos de calificación que van desde 1 punto (Nada) hasta 7 (Totalmente), conformada por las siete áreas que se presenta a continuación:

a) Búsqueda de la verdad: se refiere a la disposición para buscar la verdad, formulando interrogantes, siendo objetivo y honesto con las respuestas que se obtenga, por mucha contradicción que estas presenten.

b) Amplitud mental: alude a la disposición para poseer una mente abierta que tolere otros puntos de vista u otras opiniones divergentes.

c) Capacidad de análisis: hace referencia a la disposición a estar atento frente a situaciones que pudieran ser problemáticas, aguardando los posibles resultados o consecuencias, y valorando el empleo de la razón y el uso de pruebas, incluso frente a un problema difícil o complejo.

d) Sistemático (pensar sistemáticamente): es la disposición para la organización, la concentración, para ser capaz de enfocarse ordenadamente en una pregunta, de tal manera que no se da preferencia a ninguna forma específica de organización.

e) Confianza en el razonamiento: se refiere a la disposición para sentirse seguro de sí mismo, para fiarse de sus propias habilidades para razonar y expresar sus puntos de vista como un buen pensador.

f) Curiosidad: hace referencia a la disposición a ser indagador o estar ávido para adquirir conocimientos y aprender nuevas explicaciones.

g) Madurez para formular juicios: alude a la disposición a llevar a cabo juicios de manera reflexiva, prefiriendo aquellos que van a permitir responder los problemas, plantear interrogantes y tomar decisiones.

\section{Procedimiento}

La recolección de los datos se realizó después de solicitar los permisos y coordinaciones pertinentes, a los participantes se les informó sobre la finalidad del estudio, con el fin de que dieran su consentimiento informado y recibieron orientación adecuada para el llenado de los instrumentos. Los datos fueron procesados con el programa SPSS en concordancia con el nivel de medición de las variables y el cumplimiento de los requisitos de los estadísticos a ser utilizados para los fines de la investigación. 


\section{RESULTADOS}

Para poder determinar el estadístico a utilizar en el contraste de hipótesis, se aplicó la Prueba de Bondad de Ajuste de Kolmogorov-Smirnov a los puntajes de la Escala de Disposición hacia el Pensamiento Crítico (Escurra y Delgado, 2008a), tanto al puntaje total como a los correspondientes a sus distintas áreas (Cohen, 1988; Delgado, Escurra y Torres, 2006; Siegel y Castellan, 2012). Los resultados de la tabla 1 muestran valores $Z$ de Kolmogorov-Smirnov estadísticamente significativos tanto en los puntajes de las áreas como en el puntaje total del instrumento (búsqueda de la verdad $=.053, p<.001$; amplitud mental $=.042, \mathrm{p}<.001 ;$ capacidad de análisis $=.038, \mathrm{p}<.001 ;$ sistemático $=$ $.058, \mathrm{p}<.001$; confianza en el razonamiento $=.049, \mathrm{p}<.001$; curiosidad $=.065, \mathrm{p}<.001$; madurez para formular juicios $=.060, \mathrm{p}<.001 \mathrm{y}$ total de disposición hacia el pensamiento crítico $=.031, p<.001$ ), lo que indica que no se distribuyen acorde a la curva normal. Por lo tanto, se pueden utilizar estadísticos no paramétricos para el contraste de hipótesis, en este caso la Prueba de Kruskal-Wallis y la U de Mann Whitney.

Tabla 1

Prueba de bondad de ajuste de Kolmogorov-Smirnov para los puntajes de las áreas y del puntaje total de la Escala de Disposición hacia el Pensamiento Crítico

\begin{tabular}{lccl}
\hline Áreas & $\mathrm{M}$ & $\mathrm{D} . \mathrm{E}$. & $\mathrm{K}-\mathrm{S} \mathrm{Z}$ \\
\hline Búsqueda de la verdad & 51,59 & 5,86 & $.053^{* * *}$ \\
Amplitud mental & 51,47 & 6,56 & $.042^{* * *}$ \\
Capacidad de análisis & 51,64 & 6,83 & $.038^{* * *}$ \\
Sistemático & 53,32 & 6,96 & $.058^{* * *}$ \\
Confianza en el razonamiento & 53,27 & 6,67 & $.049^{* * *}$ \\
Curiosidad & 48,28 & 5,64 & $.065^{* * *}$ \\
Madurez para formular juicios & 52,22 & 5,96 & $.060^{* * *}$ \\
\hline Total & 361,80 & 36,82 & $.031^{* * *}$ \\
\hline
\end{tabular}

${ }^{* * *} \mathrm{p}<.001$

En la tabla 2 se presentan los resultados sobre la existencia de diferencias estadísticamente significativas en la disposición hacia el pensamiento crítico en los estudiantes de una universidad estatal de Lima Metropolitana, considerando las diversas áreas profesionales. Se observa que no se presentan diferencias estadísticamente significativas $\left(\chi^{2}=5.65 p>.05\right)$. 
Tabla 2

Comparación de los puntajes de la disposición hacia el pensamiento crítico entre áreas profesionales a través de la prueba de Kruskal Wallis

\begin{tabular}{llccc}
\hline Criterio & Áreas profesionales & $\mathrm{n}$ & $\mathrm{Rp}$ & $\chi^{2}$ \\
\hline \multirow{4}{*}{$\begin{array}{l}\text { Pensamiento } \\
\text { crítico }\end{array}$} & Ciencias de la Salud & 227 & 655,63 & \\
& Ciencias Básicas & 145 & 685,72 & \\
& Ciencias Económicas y de la Gestión & 314 & 674,01 & \\
& Humanidades y Ciencias Jurídicas y & 304 & 732,11 & \\
\hline & Sociales & & \\
\hline
\end{tabular}

${ }^{*} \mathrm{p}<.05$

En cuanto a la comparación del área búsqueda de la verdad de la disposición hacia el pensamiento crítico entre los estudiantes de una universidad estatal de Lima Metropolitana considerando las diversas áreas profesionales, los resultados (tabla 3 ) indican que no se encuentran diferencias estadísticas significativas $\left(\chi^{2}=8.08 p>.05\right)$.

Tabla 3

Comparación de los puntajes del área búsqueda de la verdad de la disposición hacia el pensamiento crítico según áreas profesionales utilizando la prueba de Kruskal Wallis

\begin{tabular}{lllll}
\hline $\begin{array}{c}\text { Pensamiento } \\
\text { crítico }\end{array}$ & \multicolumn{1}{c}{ Áreas profesionales } & $\mathrm{n}$ & $\mathrm{Rp}$ & $\chi^{2}$ \\
\hline & Ciencias de la Salud & 227 & 663,13 & \\
& Ciencias Básicas & 145 & 686,22 & \\
Búsqueda de la & Ingeniería & 386 & 690,19 & 8,08 \\
verdad & Ciencias Económicas y de la Gestión & 314 & 656,24 & \\
& Humanidades y Ciencias Jurídicas & 304 & 739,71 \\
\hline & y Sociales & 1376 & \\
\hline
\end{tabular}

${ }^{*} \mathrm{p}<.05$

En lo que se refiere a la comparación en el área amplitud mental de la disposición hacia el pensamiento crítico entre los participantes considerando las diversas áreas profesionales, los resultados en la tabla 4 muestran que no existen diferencia significativa $(\chi 2=2,998 p>.05$. 
Tabla 4

Comparación de los puntajes del área amplitud mental de la disposición hacia el pensamiento crítico según áreas profesionales utilizando la prueba de Kruskal Wallis

\begin{tabular}{lllll}
\hline $\begin{array}{l}\text { Pensamiento } \\
\text { crítico }\end{array}$ & Áreas profesionales & $\mathrm{n}$ & $\mathrm{Rp}$ & $\chi^{2}$ \\
\hline & Ciencias de la Salud & 227 & 678,74 \\
& Ciencias Básicas & 145 & 671,72 & \\
\cline { 2 - 2 } & Ingeniería & 386 & 668,48 & 2,998 \\
& Ciencias Económicas y de la Gestión & 314 & 711,91 & \\
& Humanidades y Ciencias Jurídicas y & 304 & 705,04 \\
\hline & Sociales & 1376 & \\
\hline
\end{tabular}

${ }^{*} \mathrm{p}<.05$

Con respecto al contraste análisis del área capacidad de análisis de la disposición hacia el pensamiento crítico entre los estudiantes de una universidad estatal de Lima Metropolitana considerando las diversas áreas profesionales, los resultados (tabla 5) indican que no se encuentran diferencias estadísticas significativas $\left(\chi^{2}=5.68 p>.05\right)$.

Tabla 5

Comparación de los puntajes del área capacidad de análisis de la disposición hacia el pensamiento crítico según áreas profesionales utilizando la prueba de Kruskal Wallis

\begin{tabular}{lllll}
\hline $\begin{array}{c}\text { Pensamiento } \\
\text { crítico }\end{array}$ & \multicolumn{1}{c}{ Áreas profesionales } & $\mathrm{n}$ & $\mathrm{Rp}$ & $\chi^{2}$ \\
\hline & Ciencias de la Salud & 227 & 656,40 & \\
& Ciencias Básicas & 145 & 734,13 & \\
Capacidad de & Ingeniería & 386 & 673,21 & 5,683 \\
análisis & Ciencias Económicas y de la Gestión & 314 & 681,45 & \\
& Humanidades y Ciencias Jurídicas & 304 & 717,40 & \\
& y Sociales & 1376 & & \\
\hline
\end{tabular}

${ }^{*} p<.05$

En la tabla 6 se presentan los resultados de la comparación en el área sistemática de la disposición hacia el pensamiento crítico entre los participantes, considerando las diversas áreas profesionales. Puede verse que tampoco se encuentran diferencias estadísticas significativas $\left(\chi^{2} \mathrm{de}=6,63 p>.05\right)$. 
Ana Esther Delgado, Luis Miguel Escurra, María Clotilde Atalaya, Juan Pequeña, Rolando Santiago Solís...

Tabla 6

Comparación de los puntajes del área sistemático de la disposición hacia el pensamiento crítico según áreas profesionales utilizando la prueba de Kruskal Wallis

\begin{tabular}{lllll}
\hline $\begin{array}{c}\text { Pensamiento } \\
\text { crítico }\end{array}$ & \multicolumn{1}{c}{ Áreas profesionales } & $\mathrm{n}$ & $\mathrm{Rp}$ & $\chi^{2}$ \\
\hline \multirow{3}{*}{ Sistemático } & Ciencias de la Salud & 227 & 651,99 & \\
& Ciencias Básicas & 145 & 693,37 & \\
& Ingeniería & 386 & 687,47 & 6.63 \\
& Ciencias Económicas y de la Gestión & 314 & 669,86 & \\
& Humanidades y Ciencias Jurídicas & 304 & 734,00 & \\
\hline & y Sociales & 1376 & \\
\hline
\end{tabular}

${ }^{*} \mathrm{p}<.05$

En cuanto a la comparación del área confianza en el razonamiento de la disposición hacia el pensamiento crítico entre los estudiantes de una universidad estatal de Lima Metropolitana considerando las diversas áreas profesionales, los resultados (tabla 7) indican que no existen diferencias estadísticas significativas $\left(\chi^{2}=5.85 p>.05\right)$.

Tabla 7

Comparación de los puntajes del área confianza en el razonamiento de la disposición hacia el pensamiento crítico según áreas profesionales utilizando la prueba de Kruskal Wallis

\begin{tabular}{lllll}
\hline $\begin{array}{c}\text { Pensamiento } \\
\text { crítico }\end{array}$ & \multicolumn{1}{c}{ Áreas profesionales } & $\mathrm{n}$ & $\mathrm{Rp}$ & $\chi^{2}$ \\
\hline & Ciencias de la Salud & 227 & 674,52 \\
& Ciencias Básicas & 145 & 654,09 & \\
Confianza en el & Ingeniería & 386 & 685,69 & 5,85 \\
razonamiento & Ciencias Económicas y de la Gestión & 314 & 673,76 & \\
& Humanidades y Ciencias Jurídicas y & 304 & 734,15 \\
\hline & Sociales & 1376 & \\
\hline
\end{tabular}

${ }^{*} \mathrm{p}<.05$

En lo que se refiere a la comparación en el área curiosidad de la disposición hacia el pensamiento crítico entre los participantes considerando las diversas áreas profesionales, los resultados en la tabla 8 muestran que sí existe una diferencia estadísticamente significativa, pues se obtiene un $\chi^{2}$ de 10,12. 
Tabla 8

Comparación de los puntajes del área de curiosidad de la disposición hacia el pensamiento crítico según áreas profesionales utilizando la prueba de Kruskal Wallis

\begin{tabular}{llccc}
\hline Pensamiento crítico & \multicolumn{1}{c}{ Áreas profesionales } & $\mathrm{n}$ & $\mathrm{Rp}$ & $\chi^{2}$ \\
\hline \multirow{4}{*}{ Curiosidad } & Ciencias de la Salud & 227 & 647,63 & \\
& Ciencias Básicas & 145 & 658,10 & \\
& Ingeniería & 386 & 680,84 & $10,122^{*}$ \\
& Ciencias Económicas y de la Gestión & 314 & 684,53 & \\
& Humanidades y Ciencias Jurídicas y & 304 & 747,34 \\
& Sociales & & \\
\hline
\end{tabular}

$* \mathrm{p}<.05$

Al haberse encontrado diferencias estadísticamente significativas en el área curiosidad de la disposición hacia el pensamiento crítico entre los participantes considerando las diversas áreas profesionales, de manera complementaria se estudia entre qué áreas se dan estas diferencias. En la tabla 9 se presentan los resultados de la comparación en el área curiosidad de la disposición hacia el pensamiento crítico entre los estudiantes de Ciencias de la Salud y los de Ciencias Básicas. Se observa que no hay una diferencia estadísticamente significativa $(U=16210,50, Z=-0,25, r=.01)$ entre los estudiantes de estas dos áreas.

Del mismo modo, en la tabla 9 se presentan los resultados de la comparación en el área curiosidad de la disposición hacia el pensamiento crítico entre los participantes de Ciencias de la Salud y los de Ingeniería, donde se obtiene un coeficiente $U$ de Mann Whitney de 41 646,50, un valor $Z$ de $-1,02$ y r = .04, que no es estadísticamente significativo, por lo tanto no se observa diferencia en el área curiosidad de la disposición hacia el pensamiento crítico entre los participantes de ambas áreas.

En cuanto a la comparación en el área curiosidad de la disposición hacia el pensamiento crítico entre los estudiantes de Ciencias de la Salud y los de Ciencias Económicas y de la Gestión, los resultados (tabla 9) muestran que se obtiene un coeficiente $U=33783,00$, un $Z=-1,04$ y $r=.04$, que no es estadísticamente significativo, lo que indica que ambos grupos no difieren de manera significativa.

Sin embargo, los resultados de la comparación en el área curiosidad de la disposición hacia el pensamiento crítico entre los participantes de Ciencias de la Salud y los de Humanidades y Ciencias Jurídicas y Sociales muestran un coeficiente $U$ de Mann Whitney de 29493,50, un valor $Z$ de $-2,87$ y $r=.12$, que es estadísticamente significativo y un tamaño del efecto mediano, por lo tanto se observa una diferencia estadísticamente 
significativa en el área curiosidad de la disposición hacia el pensamiento crítico entre los participantes del área Ciencias de la Salud y los del área de Humanidades y Ciencias Jurídicas y Sociales, y que son los estudiantes del segundo grupo quienes alcanzan una media de rangos más elevada.

En lo que se refiere a la comparación en el área curiosidad de la disposición hacia el pensamiento crítico entre los estudiantes de Ciencias Básicas y los de Ingeniería, los resultados en la tabla 9 muestran que no existen diferencias significativas entre ambos grupos $(U=27026,00, Z=-0,61, r=.03)$.

En cuanto a la comparación en el área curiosidad de la disposición hacia el pensamiento crítico entre los participantes de Ciencias Básicas y los de Ciencias Económicas y de la Gestión, los resultados (tabla 9) indican que se alcanzan un coeficiente $U$ de Mann Whitney de 21909,50, $Z$ de $-0,65$ y $r=.03$, que no es estadísticamente significativo, por lo tanto, no hay diferencias significativas entre los estudiantes de ambas áreas profesionales.

En la tabla 9 se presentan también los resultados de la comparación en el área curiosidad de la disposición hacia el pensamiento crítico entre los estudiantes de Ciencias Básicas y los de Humanidades y Ciencias Jurídicas y Sociales, con lo que se obtiene un coeficiente $U$ de Mann Whitney de 19199,00, un valor $Z$ de - 2,21 y r $=.10$, estadísticamente significativo y con un tamaño del efecto mediano. Esto significa que hay una diferencia estadísticamente significativa en el área curiosidad de la disposición hacia el pensamiento crítico entre los estudiantes de Ciencias Básicas y los de Humanidades y Ciencias Jurídicas y Sociales, y que son estos últimos los que alcanzan puntuaciones más elevadas.

Del mismo modo, en la tabla 9 se presentan los resultados de la comparación en el área curiosidad de la disposición hacia el pensamiento crítico entre los participantes de Ingeniería y los de Ciencias Económicas y de la Gestión, donde se observa una U = $60191,50, Z=-0,16$ y $r=.01$, lo que no es estadísticamente significativo, por lo tanto, no hay diferencias significativas entre los estudiantes de ambas áreas.

En lo referente a los resultados de la comparación en el área curiosidad de la disposición hacia el pensamiento crítico entre los estudiantes de Ingeniería y los de Humanidades y Ciencias Jurídicas y Sociales, se observa un coeficiente $U$ de Mann Whitney de 53003,50 un Z de - 2,18 y $r=.08$, que es estadísticamente significativo, y un tamaño del efecto pequeño (tabla 9), valores que indican una diferencia estadísticamente significativa en el área curiosidad de la disposición hacia el pensamiento crítico entre los estudiantes de Ingeniería y los de Humanidades y Ciencias Jurídicas y Sociales. Son los estudiantes de la segunda área los que alcanzan una media de rango mayor.

Finalmente, los resultados de la comparación en el área curiosidad de la disposición hacia el pensamiento crítico entre los participantes de Ciencias Económicas y de 
Gestión y los de Humanidades y Ciencias Jurídicas y Sociales, en la tabla 3, muestran un coeficiente $U$ de Mann Whitney de 43360.00, un valor $Z$ de $-1,97$ y $r=.08$, estadísticamente significativo y con un tamaño del efecto pequeño, lo que permite señalar una diferencia estadísticamente significativa en el área curiosidad de la disposición hacia el pensamiento crítico entre los participantes de Ciencias Económicas y de Gestión y los de Humanidades y Ciencias Jurídicas y Sociales. Los participantes de la segunda área son quienes obtienen puntajes más altos.

\section{Tabla 9}

Prueba U de Mann Whitney de los puntajes del área curiosidad de la Escala de Disposición hacia el Pensamiento Crítico de los estudiantes de diversas áreas profesionales de una universidad estatal de Lima Metropolitana

\begin{tabular}{|c|c|c|c|c|c|}
\hline Áreas profesionales & $\begin{array}{c}\text { Media de } \\
\text { rangos }\end{array}$ & $\begin{array}{c}\text { Suma de } \\
\text { rangos }\end{array}$ & $\begin{array}{l}\text { Ude Mann- } \\
\text { Whitney }\end{array}$ & Z & $\mathrm{r}$ \\
\hline CC. de la Salud & 185,41 & 42088,50 & \multirow{2}{*}{16210,50} & \multirow{2}{*}{$-0,25$} & \multirow{2}{*}{.01} \\
\hline CC. Básicas & 188,20 & 27289,50 & & & \\
\hline CC. de la Salud & 297,46 & 67524,50 & \multirow{2}{*}{41646,50} & \multirow{2}{*}{$-1,02$} & \multirow{2}{*}{.04} \\
\hline Ingeniería & 312,61 & 120666,50 & & & \\
\hline CC. de la Salud & 262,82 & 59661,00 & \multirow{2}{*}{33783,00} & \multirow{2}{*}{$-1,04$} & \multirow{2}{*}{.04} \\
\hline CC. Econom./Gest. & 276,91 & 86950,00 & & & \\
\hline CC. de la Salud & 243,93 & 55371,50 & \multirow{2}{*}{29493,50} & \multirow{2}{*}{$-2,87^{* *}$} & \multirow{2}{*}{.12} \\
\hline Human./Jurid./Soc. & 282,48 & 85874,50 & & & \\
\hline CC. Básicas & 259,39 & 37611,00 & \multirow{2}{*}{27026,00} & \multirow{2}{*}{$-0,61$} & \multirow{2}{*}{.03} \\
\hline Ingeniería & 268,48 & 103635,00 & & & \\
\hline CC. Básicas & 224,10 & 32494,50 & \multirow{2}{*}{21909,50} & \multirow{2}{*}{$-0,65$} & \multirow{2}{*}{.03} \\
\hline CC. Econom./Gest. & 232,72 & 73075,50 & & & \\
\hline CC. Básicas & 205,41 & 29784,00 & \multirow{2}{*}{19199,00} & \multirow{2}{*}{$-2,21^{*}$} & \multirow{2}{*}{.10} \\
\hline Human./Jurid./Soc. & 234,35 & 71241,00 & & & \\
\hline Ingeniería & 349,44 & 134882,50 & \multirow{2}{*}{60191,50} & \multirow{2}{*}{$-0,16$} & \multirow{2}{*}{.01} \\
\hline CC. Econom./Gest. & 351,81 & 110467,50 & & & \\
\hline Ingeniería & 330,81 & 127694,50 & \multirow{2}{*}{53003,50} & \multirow{2}{*}{$-2,18^{*}$} & \multirow{2}{*}{.08} \\
\hline Human./Jurid./Soc. & 364,15 & 110700,50 & & & \\
\hline CC. Econom./Gest. & 295,59 & 92815,00 & \multirow{2}{*}{43360,00} & \multirow{2}{*}{$-1,97^{*}$} & \multirow{2}{*}{.08} \\
\hline Human./Jurid./Soc. & 323,87 & 98456,00 & & & \\
\hline
\end{tabular}

${ }^{*} \mathrm{p}<.05^{* *} \mathrm{p}<.01$ 
En cuanto a la comparación del área madurez para formular juicios de la disposición hacia el pensamiento crítico entre los participantes considerando las diversas áreas profesionales, los resultados (tabla 10) indican que no se encuentran diferencias estadísticas significativas $\left(\chi^{2}=4,70 p>.05\right)$.

Tabla 10

Comparación de los puntajes del área formular juicios de la disposición hacia el pensamiento crítico según áreas profesionales utilizando la prueba de Kruskal Wallis

\begin{tabular}{lllll}
\hline $\begin{array}{c}\text { Pensamiento } \\
\text { crítico }\end{array}$ & \multicolumn{1}{c}{ Áreas profesionales } & $\mathrm{n}$ & $\mathrm{Rp}$ & $\chi^{2}$ \\
\hline & Ciencias de la Salud & 227 & 669,10 \\
& Ciencias Básicas & 145 & 692,92 & \\
Madurez para & Ingeniería & 386 & 714,68 & 4,70 \\
formular juicios & Ciencias Económicas y de la Gestión & 314 & 655,75 & \\
& Humanidades y Ciencias Jurídicas & 304 & 701,46 \\
& y Sociales & & \\
\hline
\end{tabular}

${ }^{*} p<.05$

En la tabla 11 se presentan los resultados de la comparación de la disposición hacia el pensamiento crítico en los estudiantes de una universidad estatal de Lima Metropolitana, considerando el sexo de los participantes. Se observa que no hay una diferencia estadísticamente significativa $(U=219169,50, Z=-1,84 p>.05)$.

Tabla 11

Comparación de los puntajes correspondientes a la disposición hacia el pensamiento crítico por sexo de una universidad estatal de Lima Metropolitana utilizando la prueba U de Mann Whitney

\begin{tabular}{cccccc}
\hline Sexo & Media de rangos & Suma de rangos & Ude Mann-Whitney & Z & $r$ \\
\hline Hombres & 704,12 & 535130,50 & \multirow{2}{*}{219169,50} & $-1,84$ & 0,05 \\
Mujeres & 664,62 & 406747,50 & & \\
\hline
\end{tabular}

${ }^{*} \mathrm{p}<.05$

En cuanto a la comparación del área búsqueda de la verdad de la disposición hacia el pensamiento crítico entre los estudiantes hombres y mujeres de una universidad estatal de Lima Metropolitana, los resultados (tabla 12) indican que no se encuentran resultados estadísticamente significativos ( $U$ de Mann Whitney $=230078,50 \mathrm{Z}=-0.34, \mathrm{p}>.05$ ). 
Tabla 12

Prueba $U$ de Mann Whitney de los puntajes del área búsqueda de la verdad de la Escala de Disposición hacia el Pensamiento Crítico por sexo de una universidad estatal de Lima Metropolitana

\begin{tabular}{cccccc}
\hline Sexo & Media de rangos & Suma de rangos & Ude Mann-Whitney & Z & $r$ \\
\hline Hombres & 689,77 & 524221,50 & & & \\
Mujeres & 682,45 & 417656,50 & 230078,50 & $-0,34$ & .01 \\
\hline
\end{tabular}

${ }^{*} \mathrm{p}<.05$

En lo que se refiere a la comparación en el área amplitud mental de la disposición hacia el pensamiento crítico entre los participantes hombres y mujeres, los resultados en la tabla 13 muestran que no existen diferencias significativas entre ambos grupos $(U=225224,50, Z=-1,01 p>.05 r=.03$.

Tabla 13

Prueba U de Mann Whitney de los puntajes del área amplitud mental de la Escala de Disposición hacia el Pensamiento Crítico por sexo de una universidad estatal de Lima Metropolitana

\begin{tabular}{cccccc}
\hline Sexo & Media de rangos & $\begin{array}{c}\text { Suma de } \\
\text { rangos }\end{array}$ & Ude Mann-Whitney & Z & $r$ \\
\hline Hombres & 676,85 & 514404,50 & 225224,50 & $-1,01$ & .03 \\
Mujeres & 698,49 & 427473,50 & & & \\
\hline
\end{tabular}

${ }^{*} \mathrm{p}<.05$

En la tabla 14 se presentan los resultados de la comparación en el área capacidad de análisis de la disposición hacia el pensamiento crítico entre los participantes hombres y mujeres, donde se obtuvo un coeficiente $U$ de Mann Whitney de 212713,00, un valor $Z$ de $-2,72$, que es estadísticamente significativo, con un tamaño del efecto pequeño $(r=.07)$. Por lo tanto, se puede concluir que en el área capacidad de análisis de la disposición hacia el pensamiento crítico se diferencian los valores promedios de los estudiantes hombres y mujeres de una universidad estatal de Lima Metropolitana y que son los hombres quienes alcanzan una media de rangos más elevada. 
Tabla 14

Prueba U de Mann Whitney de los puntajes del área capacidad de análisis de la Escala de Disposición hacia el Pensamiento Crítico por sexo de una universidad estatal de Lima Metropolitana

\begin{tabular}{cccccc}
\hline Sexo & Media de rangos & $\begin{array}{c}\text { Suma } \\
\text { de rangos }\end{array}$ & Ude Mann-Whitney & Z & $r$ \\
\hline Hombres & 712,61 & 541587,00 & 212713,00 & $-2,72^{* *}$ & .07 \\
Mujeres & 654,07 & 400291,00 & & & \\
\hline
\end{tabular}

${ }^{*} \mathrm{p}<.05$

En lo referente a los resultados de la comparación en el área sistemática de la disposición hacia el pensamiento crítico entre los participantes hombres y mujeres, se observa un coeficiente $U$ de Mann Whitney de 231069,50 y un valor $Z$ de - 0,21, que no es estadísticamente significativo (tabla 15).

Tabla 15

Prueba U de Mann Whitney de los puntajes del área sistemático de la Escala de Disposición hacia el Pensamiento por sexo de una universidad estatal de Lima Metropolitana

\begin{tabular}{cccccc}
\hline Sexo & Media de rangos & Suma de rangos & Ude Mann-Whitney & Z & $r$ \\
\hline Hombres & 688,46 & 523230,50 & 231069,50 & $-0,21$ & .01 \\
Mujeres & 684,06 & 418647,50 & & \\
\hline
\end{tabular}

${ }^{*} \mathrm{p}<.05$

En cuanto a la comparación de la existencia de diferencias estadísticas significativas en el área confianza en el razonamiento de la disposición hacia el pensamiento crítico entre los estudiantes hombres y mujeres de una universidad estatal de Lima Metropolitana, los resultados (tabla 16) indican que los valores que se obtienen $U=214462,00, Z=-2,48$ y $r=.07$ son estadísticamente significativos y el tamaño del efecto es pequeño. Asimismo, se observa que la media de rangos más elevada es obtenida por los participantes hombres $(R p=710,31)$. 
Tabla 16

Prueba U de Mann Whitney de los puntajes del área confianza en el razonamiento de la Escala de Disposición hacia el Pensamiento Crítico por sexo de una universidad estatal de Lima Metropolitana

\begin{tabular}{cccccc}
\hline Sexo & Media de rangos & Suma de rangos & Ude Mann-Whitney & Z & r \\
\hline Hombres & 710,31 & 539838,00 & & \\
Mujeres & 656,93 & 402040,00 & 214462,00 & $-2,48^{*}$ & .07 \\
\hline
\end{tabular}

${ }^{*} \mathrm{p}<.05$

En lo que se refiere a la comparación en el área curiosidad de la disposición hacia el pensamiento crítico entre los participantes hombres y mujeres, los resultados en la tabla 17 muestran que existen diferencias estadísticamente significativas entre ambos grupos $(U=196998,00, Z=-4,88$, por lo que el tamaño del efecto es mediano). Los estudiantes hombres son los que obtienen una media de rangos más alta $(733,29)$.

Tabla 17

Prueba U de Mann Whitney de los puntajes del área curiosidad de la Escala de Disposición hacia el Pensamiento Crítico por sexo de una universidad estatal de Lima Metropolitana

\begin{tabular}{cccccc}
\hline Sexo & Media de rangos & $\begin{array}{c}\text { Suma de } \\
\text { rangos }\end{array}$ & Ude Mann-Whitney & Z & r \\
\hline Hombres & 733,29 & 557302,00 & 196998,00 & $-4,88^{* * *}$ & .13 \\
Mujeres & 628,39 & 384576,00 & & \\
\hline
\end{tabular}

${ }^{* * *} \mathrm{p}<.001$

Con relación a la comparación en el área madurez para formular juicios de la disposición hacia el pensamiento crítico entre los estudiantes hombres y mujeres de una universidad estatal de Lima Metropolitana, los resultados (tabla 18) indican que se obtiene una $U=221023,50, Z=-1,58$ y $r .04$, lo que permite establecer que no son estadísticamente significativos.

Tabla 18

Prueba U de Mann Whitney de los puntajes del área madurez para formular juicios de la Escala de Disposición hacia el Pensamiento Crítico por sexo de una universidad estatal de Lima Metropolitana

\begin{tabular}{llllll}
\hline Sexo & Media de rangos & Suma de rangos & Ude Mann-Whitney & Z & $r$ \\
\hline Hombres & 701,68 & 533276,50 & 221023,50 & $-1,58$ & .04 \\
Mujeres & 667,65 & 408601,50 & & & \\
\hline
\end{tabular}

${ }^{*} p<.05$ 


\section{DISCUSIÓN}

Al analizar jerárquicamente los resultados obtenidos en las diferentes áreas de la disposición hacia el pensamiento crítico, se observa que las que alcanzan puntuaciones más altas son el área sistemática $(M=53,32$ ) y el área confianza en el razonamiento $(M=53,27)$, mientras que las que obtienen puntuaciones más bajas son el área curiosidad $(M=48,28)$, el área amplitud mental $(M=51,47)$ y el área búsqueda de la verdad $(M=51,59)$ (tabla 1$)$. Estos resultados coinciden con lo reportado por Escurra y Delgado (2008b) en universitarios de Lima y Callao, quienes encuentran que las áreas con mayores puntajes son sistemática y confianza en el razonamiento, aunque discrepan porque señalan como área con puntuaciones más altas también el área de amplitud mental. Concuerdan con los resultados de este estudio en que señalan como las áreas menos valoradas curiosidad y búsqueda de la verdad. Asimismo, los resultados obtenidos coinciden con lo señalado por Çubukcu (2006) en candidatos a maestros en Turquía, quien indica que el área con puntuaciones más bajas es curiosidad, pero discrepa en el resultado del área más valorada, porque señala que es amplitud mental. También discrepa de lo reportado por Perea (2017), quien señala que, en estudiantes del curso de Metodología de la Investigación de una universidad privada de Lima Metropolitana, las áreas de la disposición hacia el pensamiento crítico con resultados más altos fueron amplitud mental y curiosidad.

El resultado de comparar la disposición hacia el pensamiento crítico en los estudiantes de una universidad estatal de Lima Metropolitana considerando las diversas áreas profesionales mostró que no había diferencia significativa entre los grupos estudiados (tabla 2). Este resultado concuerda con lo planteado teóricamente por Facione et al. (2000) y Escurra y Delgado (2008b), quienes consideran que la disposición hacia el pensamiento crítico se refiere a la motivación interna para utilizar las habilidades del pensamiento crítico con el fin de enfrentar los problemas y tomar decisiones, por lo tanto, es de esperar que esté presente en todo estudiante universitario. Asimismo, concuerda con lo señalado por Boisvert (2004), quien plantea que el pensamiento crítico es muy importante en la vida adulta porque ayuda en las elecciones personales, tales como en la orientación profesional, la elección de un estilo de vida o la decisión sobre la adquisición de bienes materiales.

Sin embargo, el resultado obtenido en este estudio discrepa de lo reportado por Escurra y Delgado (2008b) en estudiantes universitarios de Lima y Callao, pues se encontraron diferencias estadísticamente significativas en la disposición hacia el pensamiento crítico por tipo de universidad, sexo y área profesional, y se señala que los puntajes alcanzados por los estudiantes de las áreas profesionales de Ciencias de la Salud, Ciencias Básicas (Biología, Física, Matemáticas) y Humanidades eran superiores a los de los estudiantes de las áreas de Ingenierías y Ciencias Empresariales. Consideraron los autores que las diferencias encontradas se debían a que, en las Ciencias de la Salud, 
Ciencias Básicas y Humanidades, se incentivaba y promovía más la investigación, mientras que en las áreas de Ingenierías y Ciencias Empresariales se daba prioridad a los conocimientos aplicados de tipo técnico.

En cuanto a la comparación en el área búsqueda de la verdad de la disposición hacia el pensamiento crítico entre los estudiantes de una universidad estatal de Lima Metropolitana considerando las diversas áreas profesionales (tabla 3), se encontró que no había diferencia estadísticamente significativa, lo cual coincide con lo señalado por Facione et al. (2000) acerca de que la disposición hacia el pensamiento crítico también se refiere a cómo decidir, qué hacer o creer lógicamente. El área de búsqueda de la verdad, según Escurra y Delgado (2008a), se refiere a la motivación de la persona para buscar la verdad y para hacer preguntas, siendo honesto y objetivo con las respuestas que se obtienen aun cuando estas puedan ser contradictorias, lo cual es de esperar en todo estudiante universitario.

Sin embargo, el no haber encontrado diferencias en las diversas áreas profesionales discrepa de lo encontrado por Escurra y Delgado (2008b) en estudiantes universitarios de Lima y Callao, pues ellos señalan que hallaron diferencias significativas entre las diversas áreas profesionales. Fueron los estudiantes de Ciencias de la Salud quienes alcanzaron puntuaciones más elevadas que sus pares de Ciencias Básicas (Biología, Física, Matemáticas), Ingenierías, Ciencias Empresariales y Humanidades, y los que obtuvieron las puntuaciones más bajas en el área de búsqueda de la verdad fueron los participantes del área de Ingenierías.

Asimismo, al comparar el área amplitud mental de la disposición hacia el pensamiento crítico entre los participantes considerando las diversas áreas profesionales, se observa que no hay diferencia estadísticamente significativa (tabla 4), lo cual concuerda con lo planteado por Çubukcu (2006), quien considera que el pensamiento crítico es un proceso cognoscitivo eficaz, organizado y funcional que va a permitir entender las opiniones de los demás. Por su parte, Escurra y Delgado (2008a) señalan que el área de amplitud mental hace referencia a la disposición para tener una mente abierta y tolerante a puntos de vista u opiniones divergentes.

Sin embargo, lo encontrado en la presente investigación difiere de lo reportado por Escurra y Delgado (2008b) en universitarios de Lima y Callao, pues ellos hallaron diferencias significativas entre las diversas áreas profesionales. Fueron los estudiantes de Ciencias de la Salud quienes alcanzaron puntuaciones más altas que sus pares; además, hallaron que las puntuaciones más bajas en el área de amplitud mental corresponden a los participantes de Ciencias Empresariales.

En lo que se refiere a la comparación en el área capacidad de análisis de la disposición hacia el pensamiento crítico entre los estudiantes de una universidad estatal de Lima Metropolitana considerando las diversas áreas profesionales, los resultados en la tabla 
5 muestran que no hay diferencia estadísticamente significativa, lo cual concuerda con Ennis $(1986,2011)$ y López (2012), quienes afirman que el pensamiento crítico está interesado en qué hacer o qué creer, reconociendo que la razón predomina. El área capacidad de análisis tiene que ver con la disposición a estar alerta ante situaciones potencialmente problemáticas, esperando resultados posibles o consecuencias, valorando el empleo de la razón y utilizando pruebas, incluso frente a un problema complejo o difícil (Escurra y Delgado, 2008a).

Cabe señalar que lo encontrado en la presente investigación difiere de lo hallado por Escurra y Delgado (2008b) en su investigación con universitarios de Lima y Callao: ellos encontraron diferencias significativas entre las diversas áreas profesionales. Fueron los participantes de Ciencias de la Salud los que obtuvieron puntuaciones más elevadas, y los estudiantes de Ciencias Empresariales alcanzaron las puntuaciones más bajas en lo referido a la capacidad de análisis.

Del mismo modo, con respecto al área sistemática de la disposición hacia el pensamiento crítico entre los estudiantes de una universidad estatal de Lima Metropolitana considerando las diversas áreas profesionales, se halló que no existe diferencia significativa (tabla 6). Este resultado concuerda con lo planteado por León (2014), quien confirma la existencia de ciertas disposiciones del pensamiento crítico como rasgos que caracterizan al pensador crítico, lo que es una condición propia del estudiante universitario. Del mismo modo, García et al. (2014) señalan que la práctica del buen pensamiento es esencial como expresión del área sistemática, condición presente e importante en un futuro profesional, por lo cual estaría justificada la inexistencia de diferencias según las áreas profesionales. Esto se debe a que el área sistemática, según Escurra y Delgado (2008a), constituye una característica tendiente a la disposición para la organización, al igual que para la concentración y el puntualizar ordenadamente una interrogante, sin escoger una forma determinada de organización.

Si se considera el área confianza en el razonamiento de la disposición hacia el pensamiento crítico entre los estudiantes de diversas áreas profesionales, tal como se presenta en la tabla 7, no se encontró diferencia significativa, lo que corrobora el planteamiento de Escurra y Delgado (2008b) referido a la disposición al pensamiento crítico como una motivación para afrontar situaciones problemáticas utilizando el pensamiento en la toma de decisiones. Esto es de esperar en el estudiante universitario y, por tanto, justificaría la inexistencia de diferencias significativas. Este aspecto es aún más inquietante y complejo cuando se plantea lo señalado por Boisvert (2004), que hace referencia a tres condiciones del pensamiento crítico: la exigencia de la sociedad, el aseguramiento del desarrollo socioeconómico y una producción racional sin manipulación, que se presenta de manera inherente en el universitario. Esta complejidad justificaría la inexistencia de diferencias significativas entre las áreas profesionales. Esta área de confianza 
en el razonamiento implica la seguridad del que razona para plantear sus puntos de vista de manera apropiada (Escurra y Delgado, 2008a).

Es interesante destacar que lo hallado en la presente investigación no coincide con lo que Escurra y Delgado (2008b) reportan como existencia de diferencias significativas entre los universitarios de Lima y Callao en el área confianza en el razonamiento, donde el mayor puntaje es el correspondiente a los estudiantes de Ciencias de la Salud y el menor puntaje es el obtenido por los estudiantes de Ciencias Empresariales.

En cuanto a la comparación en el área curiosidad de la disposición hacia el pensamiento crítico entre los estudiantes, los resultados muestran que existe una diferencia estadísticamente significativa entre las distintas áreas profesionales (tabla 8), por lo que fue necesario analizar de manera complementaria entre qué áreas se presentaban dichas divergencias (tabla 9). Los resultados indican que existe diferencia estadísticamente significativa en el área curiosidad de la disposición hacia el pensamiento crítico entre los participantes de Ciencias de la Salud y los de Humanidades y Ciencias Jurídicas y Sociales: los estudiantes de Humanidades y Ciencias Jurídicas y Sociales son quienes obtienen una media de rangos más elevada. Asimismo, se encontró una diferencia estadísticamente significativa entre los estudiantes de Ciencias Básicas y los de Humanidades y Ciencias Jurídicas y Sociales: nuevamente quienes alcanzan una media de rangos mayor son los estudiantes de Humanidades y Ciencias Jurídicas y Sociales. También se encontró una diferencia estadísticamente significativa en el área curiosidad de la disposición hacia el pensamiento crítico entre los participantes de Ingeniería y los de Humanidades y Ciencias Jurídicas y Sociales: en esta oportunidad también son los participantes de Humanidades y Ciencias Jurídicas y Sociales quienes obtienen una media de rangos más alta. Finalmente, se encontró una diferencia significativa entre los estudiantes de Ciencias Económicas y de la Gestión y los de Humanidades y Ciencias Jurídicas y Sociales; fue este último grupo de estudiantes el que de nuevo alcanzó la media de rangos más elevada, lo que indicaría que presenta una mayor disposición para la adquisición de conocimientos y para aprender nuevas explicaciones (Escurra y Delgado, 2008a).

Escurra y Delgado (2008b) también reportaron en el estudio realizado en universitarios de Lima y Callao que existían diferencias estadísticamente significativas entre los estudiantes de las diferentes áreas profesionales; en este caso, fueron los participantes de Ciencias de la Salud quienes obtuvieron puntuaciones más elevadas, y los estudiantes de Ciencias Empresariales alcanzaron las puntuaciones más bajas en lo referido al área curiosidad de la disposición hacia el pensamiento crítico.

En lo que se refiere a la comparación en el área madurez para formular juicios de la disposición hacia el pensamiento crítico entre los participantes de diversas áreas profesionales, en la tabla 10 se observa que no existe diferencia estadísticamente 
significativa. Tomando en cuenta lo señalado por Escurra y Delgado (2008a) - que el área de madurez para formular juicios hace referencia a la disposición para hacer juicios reflexivos para responder a los problemas, para hacer cuestionamientos y tomar decisiones es una característica esperable en todo futuro profesional-, se justificaría el hecho de no haber encontrado diferencias entre los participantes de las diferentes áreas profesionales. Sin embargo, este resultado discrepa de lo encontrado por Escurra y Delgado (2008b), quienes reportan diferencias estadísticamente significativas, con los estudiantes de Ciencias de la Salud como quienes alcanzan mayor puntaje y los de Ciencias Empresariales como quienes obtienen el menor puntaje en el área de madurez para formular juicios de la disposición hacia el pensamiento crítico.

Por otro lado, se comparó la disposición hacia el pensamiento crítico en los estudiantes de una universidad estatal de Lima Metropolitana considerando el sexo de los participantes. Los resultados muestran que no hay diferencia entre hombres y mujeres (tabla 11), lo que se justificaría si se considera lo planteado por Facione et al. (2000) y Escurra y Delgado (2008a), quienes señalan que la disposición hacia el pensamiento crítico se refiere a las características de una persona para afrontar los problemas y tomar decisiones utilizando el pensamiento. Sin embargo, este resultado discrepa de lo hallado por Escurra y Delgado (2008b), quienes reportan diferencias estadísticamente significativas en la disposición hacia el pensamiento crítico según el sexo de los participantes.

En lo que se refiere a la comparación en el área búsqueda de la verdad de la disposición hacia el pensamiento crítico entre los estudiantes hombres y mujeres de una universidad estatal de Lima Metropolitana, los resultados en la tabla 12 muestran que no hay diferencia estadísticamente significativa, lo que concuerda con lo señalado por Escurra y Delgado (2008a) en la medida en que esta área de la disposición hacia el pensamiento crítico tiene que ver con la motivación de la persona para buscar la verdad y para cuestionar, siendo honesto y objetivo con las respuestas que se obtienen aun cuando estas puedan ser contradictorias, lo que es de esperar en todo estudiante universitario con independencia del sexo. Asimismo, coincide con lo reportado por Escurra y Delgado (2008b) en estudiantes universitarios de Lima y Callao, quienes señalan que no hallaron diferencias significativas en el área búsqueda de la verdad de la disposición hacia el pensamiento crítico.

Del mismo modo, con respecto al área amplitud mental de la disposición hacia el pensamiento crítico entre los participantes hombres y mujeres, se halló que no existe diferencia significativa (tabla 13). Este resultado concuerda con lo planteado por Çubukcu (2006) y Escurra y Delgado (2008a), quienes consideran que el pensamiento crítico permite entender las opiniones que expresan los demás, y que el área amplitud mental tiene que ver con la disposición para tener una mente abierta y tolerante a otros puntos de vista u opiniones contrarias. El resultado de la presente investigación también concuerda 
con lo hallado por Escurra y Delgado (2008b) en universitarios de Lima y Callao, quienes afirman que no encontraron diferencias significativas en el área amplitud mental.

En cuanto a la comparación en el área capacidad de análisis de la disposición hacia el pensamiento crítico entre los estudiantes hombres y mujeres de una universidad estatal de Lima Metropolitana, los resultados en la tabla 14 muestran que hay una diferencia estadísticamente significativa: los hombres alcanzan una media de rangos mayor, lo que indicaría, según Escurra y Delgado (2008a), que ellos presentarían una mayor disposición a estar alertas ante situaciones problemáticas, esperando resultados posibles o consecuencias, y que valoran el uso de la razón y el empleo de pruebas, incluso frente a problemas complejos. Este resultado discrepa de lo reportado en capacidad de análisis.

Respecto de la comparación en el área sistemática de la disposición hacia el pensamiento crítico entre los participantes hombres y mujeres, los resultados muestran que no hay diferencia entre hombres y mujeres (tabla 15), lo que se justificaría si se considera lo planteado por García et al. (2014), quienes señalan que la práctica del buen pensamiento es esencial como expresión del área sistemática. Esta área, según Escurra y Delgado (2008a), tiene que ver con la disposición para la organización, para la concentración y para puntualizar de forma ordenada una interrogante, sin escoger una forma determinada de organización. Lo encontrado en esta investigación concuerda con lo hallado por Escurra y Delgado (2008b) en universitarios de Lima y Callao.

En cuanto a la comparación en el área confianza en el razonamiento de la disposición hacia el pensamiento crítico entre los estudiantes hombres y mujeres, existe una diferencia estadísticamente significativa (tabla 16): los resultados muestran que son los hombres quienes obtienen una media de rangos más elevada, lo que estaría indicando que estos estudiantes presentarían mayor disposición a tener seguridad en sí mismos, que confían en sus habilidades para razonar y expresan sus puntos de vista como buenos pensadores. Este resultado concuerda con lo reportado por Escurra y Delgado (2008b) en estudiantes universitarios de Lima y Callao, dado que también encontraron diferencias significativas en favor de los participantes hombres.

En lo que se refiere a la comparación en el área curiosidad de la disposición hacia el pensamiento crítico entre los estudiantes hombres y mujeres de una universidad estatal de Lima Metropolitana, los resultados muestran la existencia de una diferencia estadísticamente significativa (tabla 17): nuevamente son los varones quienes alcanzan una media de rangos más alta, lo que significaría que tienen mayor disposición a ser curiosos e impacientes para obtener conocimientos y explicaciones nuevas que sus pares mujeres (Escurra y Delgado, 2008a). Sin embargo, este resultado difiere de lo encontrado por Escurra y Delgado (2008b) en estudiantes universitarios de Lima y Callao, pues no hallaron diferencias significativas entre hombre y mujeres en el área curiosidad. 
Finalmente, al comparar a los participantes hombres y mujeres en el área madurez para formular juicios de la disposición hacia el pensamiento crítico, los resultados en la tabla 18 muestran que no hay una diferencia significativa, lo que se corresponde con lo planteado por Escurra y Delgado (2008a), quienes indican que el área madurez para formular juicios se refiere a la disposición para hacer juicios reflexivos al responder a los problemas, a la capacidad para hacer cuestionamientos y tomar decisiones, lo que es una característica esperable en todo universitario. Sin embargo, este resultado discrepa de lo encontrado por Escurra y Delgado (2008b), quienes reportan diferencias estadísticamente significativas según las cuales son los hombres quienes alcanzan puntuaciones más altas en el área de madurez para formular juicios.

\section{REFERENCIAS}

Alarcón, R. (2008). Métodos y diseños de investigación del comportamiento. Lima: Universidad Ricardo Palma.

Bejarano, M. Galván, F. y López, B. (2014). Pensamiento crítico y motivación hacia el pensamiento crítico en estudiantes de Psicología. Aletheia, 6(2), 202-223. Recuperado de http://aletheia.cinde.org.co/index.php/ALETHEIA/article/view/218

Boisvert, J. (2004). La formación del pensamiento crítico. México, D. F.: Fondo de Cultura Económica.

Cohen, J. (1988). Statistical power analysis for the behavioral sciences. Hillsdale: Lawrence Earlbaum Associates.

Çubukcu, Z. (2006). Critical thinking dispositions of the Turkish teacher candidates. The Turkish Online Journal of Educational Technology, 5(4), 22-36. Recuperado de www.tojet.net/volumes/v5i4.pd

Delgado, A. E., Escurra, L. M. y Torres, W. (2006). La medición en Psicología y Educación: teoría y aplicaciones. Lima: HOZLO S.R.L.

Dewey, J. (1933). How we think. Boston: D. C. Heath. Recuperado de https://ia801406. us.archive.org/17/items/howwethink000838mbp/howwethink000838mbp.pdf

Ennis, R. H. (1986). A taxonomy of critical thinking dispositions and abilities. In J. B. Baron y R. J. Sternberg (eds.), Teaching thinking skills. New York: Freeman.

Ennis, R. H. (2011). The nature of critical thinking: An outline of critical thinking dispositions and abilities. Recuperado de http://faculty.education.illinois.edu/rhennis/ documents/TheNatureofCriticalThinking_51711_000.pdf

Escurra, L. y Delgado, A. (2008a). Construcción de la escala de disposición hacia el pensamiento crítico utilizando el modelo de Samejima en alumnos universitarios de la ciudad de Lima. Teoría e Investigación en Psicología, 18(1), 41-72. 
Escurra, M. y Delgado, A. (2008b). Relación entre disposición hacia el pensamiento crítico y estilos de pensamiento en alumnos universitarios de Lima metropolitana. Persona. Revista de la Facultad de Psicología. Universidad de Lima (11), 143-175.

Facione, P. (1990). The Delphi Report, Critical Thinking: A Statement of Expert Consensus for Purposes of Educational Assessment and instruction. Millbrae: The California Academic Press.

Facione, P. A., Giancarlo, C. A., Facione, N. C. y Gainen, J. (1995). The disposition toward Critical Thinking. Journal of General Education, 44(1), 1-25. Recuperado de https:// www.insightassessment.com/content/.../file/Disposition_to_CT_1995_JGE.pdf

Facione, P. y Facione, N. (1992). The California Critical Thinking Dispossitions Inventory (CCTDI); and the CCTDI Test manual. Millbrae: California Academic Press.

Facione, P. A., Facione N. C. y Giancarlo C. A. (1997). Professional judgement and the disposition toward critical thinking. Millbrae: California Academic Press.

Facione, P. A., Facione, N. C., Giancarlo, C. A. y Gainen, J. (2000). The disposition toward Critical Thinking: Its Character, Measurement, and Relationship to Critical Skill. Informal Logic, 20(1), 61-84. ojs.uwindsor.ca/ojs/leddy/index.php/informal_logic/ article/download/2254/1698

García, I., Graterol, V. y Triviño, A. (2014). Potencialización del pensamiento: ser estratégico por medio del juego. Trabajo de grado para optar el título de Licenciatura en Pedagogía Infantil. Chía: Universidad de la Sabana. Recuperado de http:// intellectum.unisabana.edu.co/handle/10818/11199

León, F. (2014). Sobre el pensamiento reflexivo, también llamado pensamiento crítico. Propósitos y Representaciones, 2(1), 161-214. http://dx.doi.org/10.20511/pyr2014. v2n1.56

López, G. (2012). Pensamiento crítico en el aula. Docencia e Investigación (22), 41-60.

Perea, L. (2017). Disposición hacia el pensamiento crítico y su relación con el rendimiento académico en estudiantes del curso de Metodología de la Investigación de una Universidad Privada de Lima Metropolitana. Tesis para optar el grado académico de Maestro en Educación con mención en Docencia e Investigación en Educación Superior. Lima: Universidad Peruana Cayetano Heredia. Recuperado de http:// repositorio.upch.edu.pe/bitstream/handle/upch/945/Disposicion_Perea\%20 Romero\%2C\%20Lizette.pdf?sequence=3\&isAllowed=y

Sánchez, H. y Reyes, C. (2015). Metodología y diseños en la investigación científica. Lima: Business Support Aneth S.R.L.

Siegel, H. (1990). Critical Thinking as an Educational Ideal. New York: Routledge.

Sierra, J., Carpintero, E. y Pérez, L. (2010). Pensamiento crítico y capacidad intelectual. FAISCA, 15(17), 98-110. Recuperado de http://revistas.ucm.es/index.php/FAIS/ article/view/8505 
Torres, N. (2011). Influencia de las disposiciones en el desarrollo del pensamiento crítico y el aprendizaje de las Ciencias Naturales. Educar em Revista, Curitiba, Brasil [on line], 41, 247-259. Recuperado de www.scielo.br/pdf/er/n41/16.pd

Velásquez, M. y Figueroa, H. (2010). Desarrollo del pensamiento crítico en estudiantes de las carreras universitarias de mayor demanda de El Salvador. Panorama, 6(10), 7-20. Recuperado de https://dialnet.unirioja.es/descarga/articulo/4780115.pdf 